\title{
Study on Ecosystem Structure and Function of Longji Terrace
}

\author{
Xuelian $\mathrm{Zhu}^{1}$, Delan $\mathrm{Xu}^{1,}{ }^{*}$, and Kaijie Wang ${ }^{1}$ \\ ${ }^{1}$ College of Tourism \& Landscape Architecture, Guilin University of Technology, Guilin 541004, China
}

\begin{abstract}
Longji Terrace, one of the three ancient terraces in China, is a comprehensive tourist scenic spot with agricultural terraces as the main body and integrating natural landscape, cultural landscape and ethnic customs. In this paper, taking Longji Terrace in Guilin as the research object, using geographic information technology to study its ecosystem structure from the aspects of space, components, etc., to explore the material and energy flow patterns of the ecosystem, and to analyze the service functions of the ecosystem, so as to provide basic information for the healthy and sustainable development of the local ecological environment.
\end{abstract}

\section{Introduction}

With the increasing global change and human disturbance, the evolution and regulation mechanism of wetland ecosystem has become a hot spot in the world. Terrace, as one of many wetland types, was cultivated by people in old mountainous areas along the contour lines of mountains, which is not only an ancient agricultural cultural landscape, but also a heritage of human survival wisdom ${ }^{[1][2]}$. Longji Terrace National Wetland Park has beautiful natural landscape, and all the areas are ethnic minority villages. Farming labor combined with local folk customs has formed a rural landscape and farming culture with national characteristics. However, many countries and regions have developed terraced fields as an important tourism resource, and Longji terraced fields are no exception. If we do not pay attention to environmental protection and the friendly relationship between people and the environment in the development process, terraced fields will suffer some damage, which will inevitably lead to the gradual disappearance of terraced fields culture ${ }^{[3]}$.

Domestic researches on Longji terraced fields are mostly focused on ethnic tourism and farming culture, etc. ${ }^{[4][5]}$, while the research on local ecosystem is less, while the stability of ecosystem is the basis for maintaining the development of local tourism economy and the continuation of ethnic culture, and its importance is selfevident. Therefore, this paper studies various characteristics of Longji terrace ecosystem by consulting data, analyzing data and using GIS technology, and analyzes its ecological service function on this basis, which provides reference for further improving Longji terrace ecosystem environment.

\section{Materials and Methods}

\subsection{Overview of study area}

Longji Terrace is located in Longji Town, Longsheng
Autonomous County, Guilin City, Guangxi Zhuang Autonomous Region. It is located in the border of Hunan and Guangxi at the southwest foot of Yuechengling Mountains, about 76 kilometers away from Guilin city and about 30 kilometers away from county seat, and is located between 109 32' $11014^{\prime}$ E and 25 35' $2617^{\prime}$ N. Longji terraces have distinct cold and hot seasons, obvious wet and dry, large temperature difference between day and night, high altitude areas with many clouds, high humidity, frost and snow in winter, and hot summer in low altitude areas. The landform type is steep landform in erosion structure, and there are many sub-level landforms in the same large landform unit. Landform features provide a natural basis for the formation of terraces, and the terraced fields in China are large in scale. Terraces are distributed between 300 and 1,100 meters above sea level, with a vertical height difference of $800 \mathrm{~m}$ meters. The slopes of terraces are mostly between 26 and 35 degrees, and the maximum slope is 50 degrees. Most of the soil in the terraced fields is sandstone and shale, and a small amount is mountainous thin soil developed from granite, limestone and quartzite parent materials; The vertical distribution of soil is obvious. The soils in terraced fields are mainly red soil, yellow red soil and yellow soil. Most of them are mountainous red soil below $500 \mathrm{~m}$, yellow red soil at $500 \sim 800 \mathrm{~m}$, yellow soil above $800 \mathrm{~m}$ and yellow brown soil above $1350 \mathrm{~mm}$. The $\mathrm{pH}$ value is generally between 4.4 and 6.5 , and the thin soil layer is the most, and the topsoil is rich in organic matter, and the soil is fertile, which provides better conditions for plant growth.

Longji Scenic Area covers an area of 70.1 square kilometers, with contiguous terraced fields covering an area of 10,734 mu. Longji terraced fields are divided into Jinkeng (Dazhai) Yao terraced fields scenic spot and Ping 'an Zhuang terraced fields scenic spot. There are two ethnic groups living in the scenic spot, Zhuang and Yao, mainly Zhuang, and Zhuang in Longji is the representative

\footnotetext{
$\overline{{ }^{*} \text { Corresponding author: lotus921@126.com }}$
} 
of Northern Zhuang. The villages distributed in the scenic area include Ping 'an Zhuang Village, Longji Ancient Zhuang Village, Huangluoyao Village and Jinkeng Dayao Village. Ping 'an Zhuang Village has 170 households with a population of more than 700 , which is a Zhuang community with Liao surname; There are more than 200 households and more than 1,000 people in Longji Ancient Zhuang Village, which is a Zhuang community with three surnames of Pan, Liao and Hou; Huangluo Yaozhai has 60 households with a population of more than 400 , which is a Yao inhabited area with the surname Pan. There are 245 families in Dayao Village in Jinkeng, with a population of more than 1,130, which is a Yao inhabited area with the surname Pan.

\subsection{Data collection and processing}

This paper takes Longji Terrace Scenic Spot as the research object, and uses geographic information technology to extract its land use, contour line and river network distribution, studies the scenic spot structure and material and energy flow, and then extracts the ecological service function of this area.

The land use data used in this paper comes from the global land cover data product GlobeLand30 in 2020, which is used to extract the digital elevation map of contour lines and river networks. the data comes from ASTER GDEM V2 of geospatial data cloud, with a spatial resolution of $30 \mathrm{~m}$.

\section{Structure of Longji Terrace Ecosystem}

\subsection{Space structure}

Longji Terrace Scenic Area is a terraced group which mainly consists of Ping 'an Zhuang Terrace and Jinkeng Hongyao Terrace and radiates to surrounding villages. The scenic area covers a total area of 70.1 square kilometres, with contiguous terraced fields covering an area of 10,734 mu, mainly inhabited by Zhuang and Yao people. In 2017, there were 773 households with 3,354 people in Dazhai Village, Ping 'an Village and Longji Village.

As shown in Figure 1, the top of Longji terraced fields is forest, the middle part is terraced fields, and the lower part is river. The land use types in the study area are mainly cultivated land and woodland, including paddy fields and dry land, while woodland includes woodland and sparse woodland. In the whole ecosystem of Longji terraced fields, ethnic minorities live in harmony with the local natural environment, and human activities vertically divide the mountain in Longji area into three parts: forest, village, terraced fields and rivers. Forests can conserve water, and accumulate domestic water for people by collecting rivers at the bottom through plant interception and surface runoff; People solve the food problem through terraced farming. The spatial distribution of forest-villageterrace-river constitutes an ecosystem suitable for local residents' production and life. Its vertical spatial distribution is shown in Figure 2.

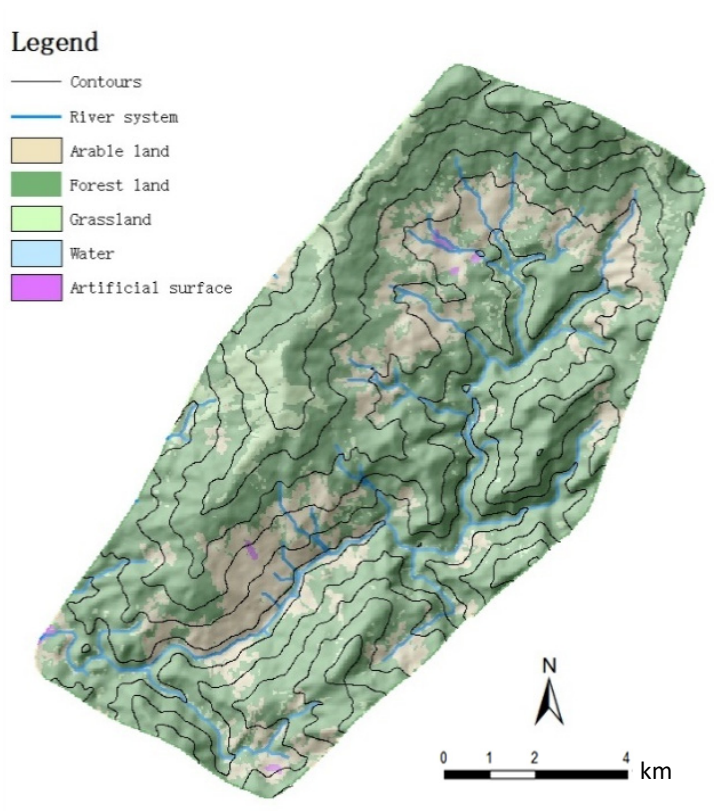

Fig. 1. Land Use Distribution of Longji Terrace

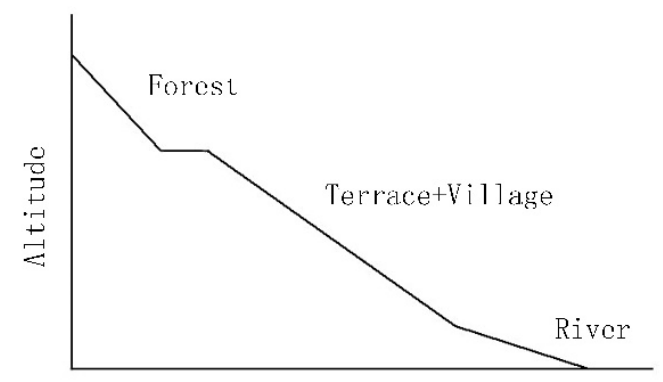

Fig. 2. Vertical Spatial Distribution of Longii Terrace

\subsection{Composition structure}

\subsubsection{Terrace ecosystem}

The composition structure of Longji terrace ecosystem mainly includes producers, consumers and decomposers. Producers are crops growing in terraces and herbaceous plants on ridges, and there are also fish and loach in the position of secondary producers, and consumers are human beings and domestic animals, among which poultry are chickens, ducks and geese, domestic animals are cattle, pigs and sheep, and decomposers are various soil microorganisms and bacteria.

Primitive cultivated japonica rice appeared in Nanling Mountain where Longsheng is located from 6000 to 12000 years ago, which is one of the cradles of artificially cultivated rice in the world. During the Qin and Han Dynasties, terraced farming was formed in Longsheng ${ }^{[6]}$. Longsheng terraces were developed on a large scale in Tang and Song Dynasties, and basically reached the existing scale in Ming and Qing Dynasties. Longji Terrace has a history of at least 2300 years, which can be called the hometown of terraced fields in the world ${ }^{[7]}$. The ancestors of Zhuang and Yao people gave full play to their wisdom and fighting spirit, and turned one hillside after 
another, which was originally densely forested, into a terraced field community, which was layered on top of the mountain.

\subsubsection{Natural ecosystem}

The vegetation of forest ecosystem in Longji area belongs to mid-subtropical evergreen vegetation, and there are more than 1,000 species of tree, shrub and herb. Affected by topography and climate, vegetation in the system is distributed vertically and grows luxuriantly. There are 304 species of vascular plants in Longji Terrace Scenic Area. Among them, there are 19 species of ferns, 9 species of gymnosperms and 276 species of angiosperms. At an altitude of 800-1300 m, evergreen and deciduous broadleaved mixed forest is the main forest, supplemented by economic forests such as pine and fir, and at an altitude of 300-800 m, China fir (Cunninghamia lanceolata (Lamb.) Hook.), masson pine (Pinus massoniana Lamb), camellia oleifera (Camellia oleifera), tung tree (Vernicia fordii (Hemsl.) Airy Shaw), bamboo (Phyllostachys heterocycle (Carr.) Mitford cv.Pubescens) and many broad-leaved forest species are present. Abundant precipitation ensures the main source of water in the ecosystem. The precipitation is filtered by the forest on the top of the mountain, or infiltrates the soil, or gathers into streams, flows through villages and terraces, and finally flows into the valley Jinjiang River. There are 112 species of vertebrates in Longji Terrace Scenic Area. Among them, there are 13 species of fish, 7 species of amphibians, 10 species of reptiles, 58 species of birds and 14 species of mammals. Most of them are birds and amphibians, among which birds are the most, with 58 species.

\subsection{Material circulation and energy flow in ecosystem}

Material and energy flow is the core of ecosystem. The specific spatial structure of Longji Terrace makes its ecosystem form a unique way of material and energy flow, and its medium of material and energy flow includes water, nutrients and microorganisms. The forest ecosystem in Longji Terrace Scenic Area is vast in area and complex in structure. According to previous studies, the annual fixed solar energy of forest ecosystem is about $3.1 \times 1017 \mathrm{~kJ}$, accounting for $63 \%$ of the annual fixed solar energy of terrestrial ecosystem ${ }^{[8]}$. Therefore, forest ecosystem is the energy base of Longji area. After receiving solar energy, forest vegetation is converted into available organic matter and energy, which passes through villages and is strengthened by human and animal behaviours, then flows into terrace system, where it is used by crops and soil organic matter layer by layer, and finally flows into valleys and rivers. The space formed based on this mountain structure layout constitutes a relatively complete mountain agriculture and forestry ecosystem. In this kind of ecosystem, the basic material circulation and energy flow needed by ecology can be well completed, and the basic vitality and ecological balance of the ecosystem can be maintained, that is, the input and output of energy and material in the system are basically equal ${ }^{[9]}$, which makes the life and production of Longji people stable, and the ecological environment has not been destroyed due to tourism development.

\section{Function of Longji Terrace Ecosystem}

\subsection{Providing agricultural products}

The most basic function of Longji terrace ecosystem is to provide rice yield. Rice is an annual gramineous plant with a height of about $1 \mathrm{~m}$, which has no strict requirements on the growth environment and can grow better under the conditions of high temperature, high humidity and short sunshine. The most important food crop in China is rice. Rice planted in terraced fields provides the main product for human beings, followed by by by-products such as straw, rice husk and rice bran. Besides being used as food source for residents, rice can also be bought and sold to realize economic value, and straw, rice husk and rice bran can also be widely used in industrial and agricultural production ${ }^{[10]}$. Terraced fields maintain the life of residents of all ethnic groups in the ecosystem, and derive economic and tourism value.

\subsection{Conservation of both moisture and soil}

The position of forest in ecosystem is very important. When rainfall occurs, the humus decomposed by dead branches and fallen leaves in the forest on the top of the mountain can reduce the runoff of water, make most of the rainwater seep into the soil layer, and conserve water effectively. The forest on the top of Longji area provides abundant water sources for villages and terraces. The water flows from top to bottom, and besides meeting people's living and production needs, the water flows into the Jinjiang River under the mountain. Paddy field is also a huge "reservoir". In the rainy season, the ridge can intercept rainwater for people to use.

There are few mountains and lots of land in Longji area, and terraced fields are built along the mountain shape. In order to make full use of land resources, some terraced fields are only a few square meters. The terraced terrain slows down the speed of water erosion, and the rice ridge also intercepts some sediment and humus. The accumulation of humus can enhance soil fertility and improve soil quality. Planting rice in terraced fields can effectively prevent soil erosion caused by rainfall erosion and play a very important role in improving soil environment.

\subsection{Maintaining biodiversity}

The mountainous spatial layout of Longji terraced field ecosystem and superior phenological conditions have created a wide variety of local animal and plant resources with distinctive characteristics, which have been maintained up to now. Stable biodiversity has become an important carrier of agricultural cultural heritage. Strengthening the protection of natural environment and 
ecosystem where terraced fields are located is conducive to better enriching biodiversity.

\subsection{Forming a self-cleaning system}

Clean spring water in Longji terrace ecosystem can be directly introduced by local villagers and used in daily life and production, and the generated domestic sewage, faeces and garbage can be transported to terraces with natural or artificial drainage systems and received by terraces along the mountain, thus increasing the soil fertility of paddy fields ${ }^{[11]}$. Sewage and so on can reduce the pollution of Jinjiang River at the bottom after being filtered by terraced fields. In this way, the ecosystem forms a natural self-purification system, which plays a positive role in the protection of nature and human settlements.

\subsection{Humanistic value}

Terrace is an ancient and important agricultural cultural landscape, and it is the heritage of human survival wisdom. Its formation is integrated with human conscious intervention and regulation. Many years ago, people in mountainous areas left behind this farming method. During the long-term farming of terraced fields, local landscapes and local cultures with local characteristics were formed. In the long history of human agricultural development, rich rice culture connotations of terraced fields were accumulated, and rice culture with special value was formed. The natural landscape of Longji terraced fields is beautiful and rich, and the area is full of ethnic minority villages. Farming labour often combines the folk customs of local Zhuang and Yao people, forming a farming culture with national characteristics. The adaptation and combination of natural environment and folk culture is the crucial reason for the maintenance of Longji terrace ecosystem.

\section{Conclusion and outlook}

The spatial structure of forest-village-terrace-river in Longji terrace ecosystem makes the system filter water and nutrients layer by layer and fully utilize and absorb them. The ecological environment composed of terraces, forests and rivers provides the villagers with the background of life and the foundation of survival. Terrace ecosystem has significant benefits in ensuring food supply, conserving water and soil, conserving water, improving soil fertility, maintaining ecological balance and preserving historical memory. On the one hand, Longji terraced field ecosystem should retain and continue to play its existing advantages, on the other hand, there are some ecological and development worries in this system:

(1) The villagers' ecological awareness is weak, they don't know the service value of terrace ecosystem well, and they don't give full play to terrace ecological function; Tourism development has also broken the ecological environment, which is not conducive to the sustainable development of terrace ecosystem, so it is necessary to enhance the villagers' awareness of ecological protection.

(2) With the development of tourism in Longji area, the number of villagers participating in tourism business is increasing, and people's enthusiasm for planting rice is low, so they can't take agriculture as the main economic source, and food security and yield can't be guaranteed.

(3) The number of young and middle-aged migrant workers in rural areas is increasing, farmers are older, and the difficulty of farming is increasing. Terraces with complex terrain are inevitably abandoned, which affects the stability of ecosystem and hinders the development of terrace ecology, tourism and economy.

Based on the above, we can think that up to now, Longji Terrace Scenic Area has a relatively complete ecosystem integrating human and nature, but it is necessary to strengthen the control and control in tourism development and vegetation resources management, and rationally develop and rectify it to meet the requirements of sustainable development of the scenic area. In the future, we can continue to study the ecosystem in this area from the aspects of biological species composition, quantitative changes or other spatio-temporal changes.

\section{Acknowledgments}

Supported by: Research and Demonstration of Key Technologies for Sustainable Utilization of Karst Landscape Resources in the Lijiang River Basin (2019YFC0507500)

\section{References}

1. J. Y. Tian, JSU, (2020)

2. H. Y. Lu, X. Z. Yuan, X. F. Wang, CJE, 38, 18901899(2019)

3. L. Chen, GS, 55, 153-154(2021)

4. X. F. Xie, J. J. Liu, JGST, 232-234(2018)

5. Y. Lu, AMA, 85-95(2018)

6. Z. J. Wu, J. Luo, GES, 117-127(2020)

7. T. T. Tang, GXU, (2019)

8. Y. Z. Cui, FID, 31-32(2013)

9. H. P. Zhang, C. M. Zhu, D. S. Yang, SWCC, 4950(2011)

10. H. Y. Sun, F. B. Li, F. P. Fang, JAAS, 39, 1718917191(2011)

11. W. J. Zheng, B. B. Zhang, Z. J. Wu, CLA, 35, 2024(2019) 